Title:

Solving Phase Appearance/Disappearance Two-Phase Flow Problems with High Resolution

Staggered Grid and Fully Implicit Schemes by Jacobian-Free Newton-Krylov Method

Author names and affiliations:

Ling Zou, Ph.D.

Haihua Zhao, Ph.D.

Hongbin Zhang, Ph.D.

Idaho National Laboratory, P.O. Box 1625, Idaho Falls, ID 83415-3870 USA

Corresponding author:

Ling Zou,

Email: $\underline{\text { ling.zou@inl.gov }}$

Phone: 1-208-526-7204

Fax: 1-208-526-2930

Address: Idaho National Laboratory, P.O. Box 1625, Idaho Falls, ID 83415-3870 USA 


\title{
Solving Phase Appearance/Disappearance Two-Phase Flow Problems with High Resolution Staggered Grid and Fully Implicit Schemes by Jacobian-Free Newton-Krylov Method \\ Ling Zou ${ }^{1}$, Haihua Zhao, and Hongbin Zhang \\ Idaho National Laboratory, P.O. Box 1625, Idaho Falls, ID 83415-3870 USA
}

\begin{abstract}
The phase appearance/disappearance issue presents serious numerical challenges in two-phase flow simulations. Although many existing nuclear reactor safety analysis codes use different kinds of treatments for the phase appearance/disappearance problem, there are no fully consensual solutions. In this work, a high-resolution spatial discretization scheme on staggered grid mesh and fully implicit time integration methods were used in two-phase flow simulations. Numerical treatments of the momentum equations were introduced for the disappearing phase without the need to use a cut-off value on void fraction. Discretized nonlinear system was solved using the Jacobian-free Newton Krylov (JFNK) method, which does not require expensive and error-prone derivation and implementation of analytical Jacobian matrix. These methods were tested with several two-phase flow problems with phase appearance/disappearance phenomena considered, such as a linear advection problem, an oscillating manometer problem, and a sedimentation problem. The combined methods demonstrated extremely robust and stable behaviors in solving the one-dimensional two-phase flow problems with phase appearance/disappearance. High-resolution spatial discretization and second-order fully implicit method also demonstrated their capabilities in significantly reducing numerical errors.
\end{abstract}

Keywords: Two-Phase Flow, Jacobian-Free Newton-Krylov method, High-resolution spatial discretization scheme, Phase disappearing; Implicit

\footnotetext{
${ }^{1}$ Corresponding author, Email: ling.zou@inl.gov
} 


\section{INTRODUCTION}

In the nuclear engineering field, two-phase flow is an important phenomenon closely related to the normal operations and accident conditions of nuclear reactors. Accurate modeling and simulation of one-dimensional (1-D) two-phase flow are critical to the safety analyses of nuclear reactors. During the last four decades, several nuclear reactor system analysis codes had been developed to solve the 1-D two-phase flow equations to represent the complex reactor systems. These codes, such as RELAP5 [1] and TRAC [2], have gained great successes in supporting reactor safety analyses, as well as design and licensing of new reactors. Low order spatial discretization scheme (such as first-order upwind method) and operator-splitting (OS) type of time integration schemes (such as semi-implicit method) were widely used in these codes. It is well understood that these low-order numerical schemes generally introduce excessive numerical errors. As new reactor designs emerge, there are new challenges appearing in numerical simulations of reactor systems, such as long transient and strongly coupled multi-physics problems. As we aim to improve the numerical accuracy of reactor safety analysis codes, it is important to consider advanced numerical schemes and methods in the development of nextgeneration analysis codes, such as: 1) high-resolution spatial discretization scheme in order to improve spatial accuracy; 2) fully implicit time integration schemes in order to allow larger time steps to be used and to improve temporal accuracy; and 3) advanced solving method (such as the Jacobian-free Newton-Krylov method) to efficiently solve the highly nonlinear system.

The phase appearance/disappearance issue presents serious numerical challenges in the two-phase flow simulations. In literatures, there are few works directly dealing with such a topic. Many existing reactor safety analysis codes use different kinds of treatments for the phase appearance/disappearance problem. However, to our best knowledge, there are no fully consensual solutions. For example, in RELAP5-3D phase disappearance treatment, if the calculated void fraction is less than zero, the void fraction is reset to zero. If the calculated void 
fraction is much less than zero, the solver fails and the time step is reduced [3]. The system analysis code, CATHARE, is one of the rare exceptions that employed a fully implicit scheme (as one of its three time integration schemes) [4]. The strategy that the CATHARE code used to deal with numerical difficulties associated with the phase appearance/disappearance phenomenon is described by Bestion in [5]. When the void fraction tends to the prescribed minimum value of $\alpha_{\min }=10^{-5}$ or the maximum values of $\alpha_{\max }=1-10^{-6}$, the interfacial mass and energy transfers are conditioned so that the predicted void fraction does not exceed these limiting values [5].

In a recent study from Cordier et al. [6], it was pointed out that the numerical challenges of simulating the phase appearance/disappearance phenomena in two-phase flow are multifold: the loss of hyperbolicity of the equation system when a phase appears or disappears, and the lack of positivity of standard shock capturing schemes. In a recent study done by Ashrafizadeh et al. [7], a Jacobian-free Newton Krylov (JFNK) method was used to solve 1-D two-phase flow problems with implicit time integration scheme. An extended AUSM+ scheme and the phase appearance/disappearance treatment strategy, originally proposed in [8], were adapted in that work. However, the same phase appearance/disappearance treatment strategy, which has been proved to be effective for explicit time integration scheme, did not give satisfactory results in their JFNK and implicit schemes. For example, void fraction smaller than $10^{-3}$ is set to be $10^{-3}$. Frepoli et al. [9] made an attempt to implicitly solve the 1-D three-field two-fluid two-phase flow equations using a Newton's method. Special treatment was proposed for the phase appearance/disappearance problem, of which the key concept is to modify the momentum equation of the vanishing phase in order to make it not singular.

In recent years, the JFNK method has gained many successes in solving large-scale nonlinear systems in different disciplines (a good review could be found in [10]). An attractive feature of the JFNK method is that the expensive evaluations of Jacobian matrix could be avoided. In 
addition, the analytical derivation of Jacobian matrix could be cumbersome, time consuming and error-prone, especially when many empirical correlations used in the thermal-hydraulics field have complicated forms. Mousseau had done several pioneering works using the JFNK method to solve the coupled two-phase flow and heat conduction problem [11-14], as well as coupled twophase flow and reactor physics problem [15]. His work provided important insights on time step control, preconditioning scheme for JFNK method in solving two-phase flow problems. However, many challenging issues of the two-phase problems, such as the phase appearance/disappearance issue, were not addressed. Ashrafizadeh's work [7] is another example of the JNFK applications in solving two-phase flow problems, as we have mentioned in the previously.

The main objective of this study is to apply high-resolution spatial discretization and high-order implicit time integration scheme in the framework of JFNK method in solving two-phase flow problems with phase appearance/disappearance phenomena considered. In this study, we limit our efforts on phase appearance/disappearance problems caused by phase advection. The boiling and condensation induced phase appearance/disappearance phenomena will be investigated in our future study. In the following sections, the simplified single-pressure two-fluid two-phase flow model, commonly used in the reactor safety analysis codes, will be presented. The high-resolution spatial discretization scheme on staggered grids, implicit time integration schemes, and JFNK method will be briefly discussed for the purpose of completeness. Several hydrodynamics twophase flow problems were chosen to demonstrate the advantages of using high-resolution spatial discretization and high-order implicit time integration schemes, as well as the robustness of using the JFNK method as the nonlinear solver.

\section{1-D TWO-FLUID TWO-PHASE FLOW MODEL}

The two-fluid single pressure two-phase flow equations used in this work are similar to those used in the existing system analysis codes, such as RELAP5 [1], TRAC [2], and CATHARE [16]. 
In this study, efforts are focused on the hydrodynamic benchmark problems, and thus the two energy equations are not included. In addition, the high-resolution spatial discretization scheme applied on a staggered grid mesh does not require the equation system to be hyperbolic. Therefore, additional terms to render the equations system to be hyperbolic, such as the virtual mass term in the RELAP5 code [3] and $p_{i} \nabla \alpha$ term in the CATHARE code [16], are not included. In addition, without considering the mass transfer between the two phases and wall friction, the six-equation system commonly used in existing reactor system codes is further reduced to a fourequation system, including two mass equations and two momentum equations,

$$
\begin{gathered}
\frac{\partial\left[(1-\alpha) \rho_{l}\right]}{\partial t}+\frac{\partial\left[(1-\alpha) \rho_{l} u_{l}\right]}{\partial x}=0 \\
\frac{\partial\left(\alpha \rho_{g}\right)}{\partial t}+\frac{\partial\left(\alpha \rho_{g} u_{g}\right)}{\partial x}=0 \\
\frac{\partial u_{l}}{\partial t}+u_{l} \frac{\partial u_{l}}{\partial x}+\frac{1}{\rho_{l}} \frac{\partial p}{\partial x}-g_{x}-\frac{C_{i}}{(1-\alpha) \rho_{l}}\left(u_{g}-u_{l}\right)\left|u_{g}-u_{l}\right|=0 \\
\frac{\partial u_{g}}{\partial t}+u_{g} \frac{\partial u_{g}}{\partial x}+\frac{1}{\rho_{g}} \frac{\partial p}{\partial x}-g_{x}-\frac{C_{i}}{\alpha \rho_{g}}\left(u_{l}-u_{g}\right)\left|u_{g}-u_{l}\right|=0
\end{gathered}
$$

in which, the subscripts $l$ and $g$ denote the liquid phase and the gas phase, respectively. The variables to be solved from this set of equations are: $\boldsymbol{U}=\left[p, \alpha, u_{l}, u_{g}\right]^{\mathrm{T}}$, which are pressure, void fraction (volume fraction of the gas phase), liquid phase velocity, and gas phase velocity, respectively. Linearized barotropic equations of state are used in this study for both phases: 


$$
\begin{gathered}
\rho_{l}(p)=\rho_{l, 0}+\frac{1}{\left(\frac{d p}{d \rho_{l}}\right)_{0}}\left(p-p_{0}\right) \\
\rho_{g}(p)=\rho_{g, 0}+\frac{1}{\left(\frac{d p}{d \rho_{g}}\right)_{0}}\left(p-p_{0}\right)
\end{gathered}
$$

in which, $\rho_{l, 0}=10^{3} \mathrm{~kg} / \mathrm{m}^{3}, \rho_{g, 0}=0.5 \mathrm{~kg} / \mathrm{m}^{3}$, and $p_{0}=10^{5} \mathrm{~Pa}$, are reference liquid phase density, reference gas phase density, and reference pressure, respectively. $\left(\frac{d p}{d \rho_{l}}\right)_{0}=10^{7} \mathrm{~Pa} /\left(\mathrm{kg} / \mathrm{m}^{3}\right)$ and $\left(\frac{d p}{d \rho_{g}}\right)_{0}=10^{6} \mathrm{~Pa} /\left(\mathrm{kg} / \mathrm{m}^{3}\right)$ reflect the compressibility of the liquid phase and gas phase, respectively.

For the interfacial drag term in the momentum equations, a simplified interfacial drag model was used, similar to the model used in Städtke's work [17],

$$
C_{i}=\frac{1}{8} C_{d} a^{i n t} \rho_{m}
$$

in which, the interfacial area density is estimated as,

$$
a^{i n t}=\frac{3 \alpha(1-\alpha)}{r_{p}}
$$

the mixture density is defined as,

$$
\rho_{m}=\alpha \rho_{g}+(1-\alpha) \rho_{l}
$$

and, $C_{d}$ is the drag coefficient, $r_{p}$ is the particle size. In this work, constant values, 0.44 for $C_{d}$, and $0.5 \times 10^{-3} m$ for $r_{p}$ were used, the same as in [17]. These simplified equations of states, closure models, and parameters will give qualitatively correct answers, and they will not affect our numerical investigations on the phase appearance/disappearance problems. However, to achieve more realistic results in the simulations of two-phase flow, more complex interfacial drag model is necessary. 


\section{NUMERICAL AND SOLUTION METHODS}

In this section, we will briefly discuss: 1) the high-resolution spatial discretization scheme based on staggered grid mesh arrangement, 2) several fully implicit time integration schemes, and 3) the JFNK method in solving the nonlinear equation system.

\subsection{High-resolution spatial discretization with staggered grids}

In this work, we have successfully extended a high-resolution spatial discretization scheme on staggered grid mesh to solve the 1-D two-phase flow problems. The original scheme was developed to solve shallow water problems, by Stelling and Duinmeijer [18]. There are many high-resolution schemes developed using co-located meshes, in this work the staggered grid mesh is used for two reasons: 1) it is flexible to handle the two-phase equations in the primitive forms; and 2) many existing nuclear reactor analysis codes are using such staggered grid mesh (for backward compatibility purpose). The high-resolution scheme was obtained by introducing the linear reconstruction of the variable solutions and slope limiter into the original first-order upwind. For the purpose of completeness, the spatial discretization scheme is briefly discussed in this subsection.

For the staggered grid mesh commonly used in existing reactor safety system codes, scalar variables (such as pressure and density) are arranged in cell centers, while vector variables (such as velocity) are arranged on cell edges.

$$
\left.\frac{\partial\left(\alpha_{g} \rho_{g} u_{g}\right)}{\partial x}\right|_{i}=\frac{1}{\Delta x}\left[\left(\alpha_{g} \rho_{g} u_{g}\right)_{i+1 / 2}^{*}-\left(\alpha_{g} \rho_{g} u_{g}\right)_{i-1 / 2}^{*}\right]
$$

and

$$
\left(\alpha_{g} \rho_{g} u_{g}\right)_{i+1 / 2}^{*}=u_{g, i+1 / 2}\left\{\begin{array}{l}
\alpha_{g, i} \rho_{g, i} \\
\alpha_{g, i+1} \rho_{g, i+1}
\end{array} \quad \text { if } \begin{array}{cc}
u_{g, i+1 / 2}>0 \\
\text { otherwise }
\end{array}\right.
$$

The discretization of the advection term in the momentum equations is done in a similar way, 


$$
\left.u_{g} \frac{\partial u_{g}}{\partial x}\right|_{i+1 / 2}=\frac{1}{\Delta x} u_{g, i+1 / 2}\left\{\begin{array}{lr}
u_{g, i+1 / 2}-u_{g, i-1 / 2} & \text { if } \begin{array}{r}
u_{g, i+1 / 2}>0 \\
u_{g, i+3 / 2}-u_{g, i+1 / 2}
\end{array} \text { otherwise }
\end{array}\right.
$$

The high-resolution version of the discretization is obtained by replacing the donor values with linearly reconstructed cell edge values. Equations (10) and (11) become,

-..-- Piecewise linear reconstruction using slope limiter

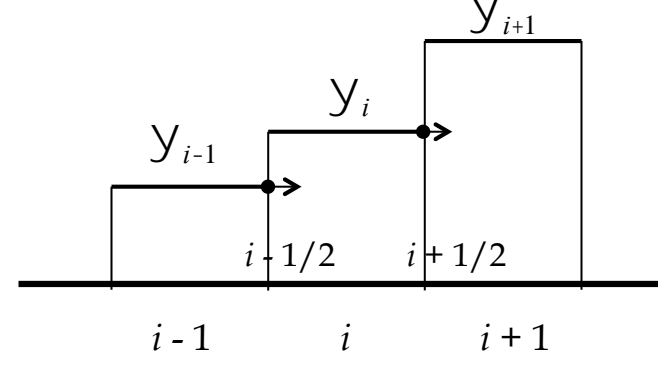

(a) First-order, scalar variables

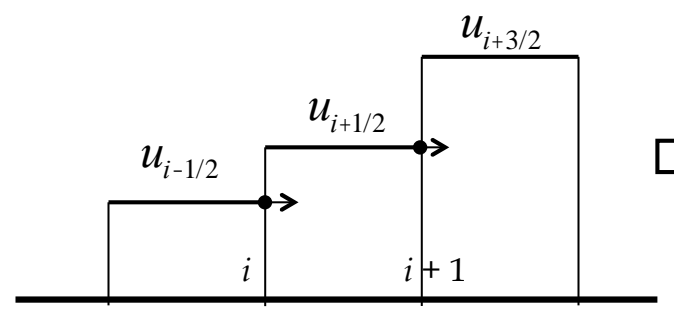

$i-1 / 2 \quad i+1 / 2 \quad i+3 / 2$

(c) First-order, velocity

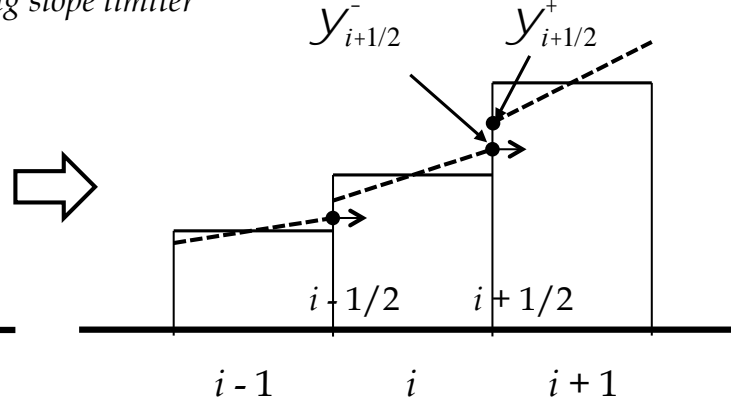

(b) High-resolution, scalar variables

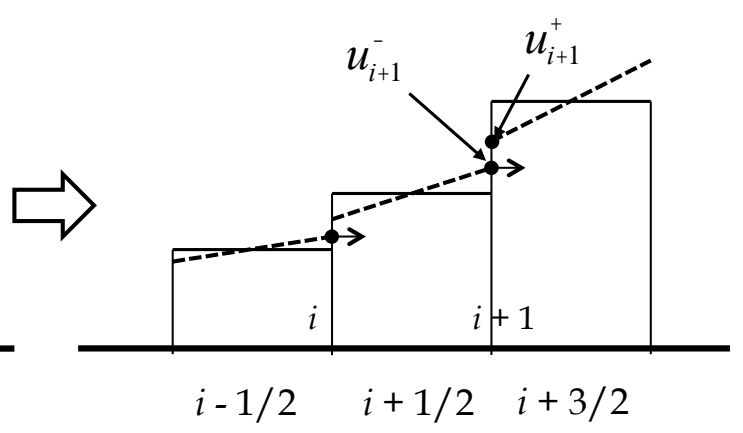

(d) High-resolution, velocity

Figure 1. Schematic illustration of the first-order and high-resolution spatial discretization schemes for the staggered grid mesh. Positive velocities are assumed in this plot. $\psi$ represents scalar properties (such as pressure and void fraction), and $u$ is velocity.

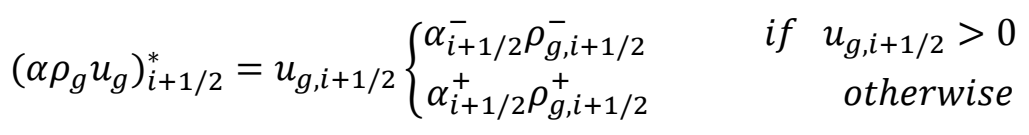

and 


$$
\left.u_{g} \frac{\partial u_{g}}{\partial x}\right|_{i+1 / 2}=\frac{1}{\Delta x} u_{g, i+1 / 2}\left\{\begin{array}{lr}
u_{i+1}^{-}-u_{i}^{-} \\
u_{i+1}^{+}-u_{i}^{+}
\end{array} \quad \text { if } \begin{array}{r}
u_{g, i+1 / 2}>0 \\
\text { otherwise }
\end{array}\right.
$$

in which, the superscript '+' and '-' denote the reconstructed values (Figure 1). The linear reconstruction of variables, such as $\alpha, p$, and $u$, can be done by any standard slope limiter schemes, such as the symmetric Van Albada [19] limiter used in this work,

$$
\phi(r)= \begin{cases}\frac{r+r^{2}}{1+r^{2}} & \text { otherwise } \\ 0 & \text { if } r \geq 0\end{cases}
$$

in which, $r$ is the nonlinear indicator. Using pressure as an example, at the $i^{\text {th }}$ cell, the indicator is defined as, $r=\left(p_{i}-p_{i-1}\right) /\left(p_{i+1}-p_{i}\right)$. The reconstructed slope can then be calculated as,

$$
\left(p_{x}\right)_{i}=\frac{1}{\Delta x} \phi(r)\left(p_{i+1}-p_{i}\right)
$$

\subsection{Numerical treatment with phase disappearance}

When one of the two phases is absent, source terms in the momentum equations need proper numerical treatment to avoid numerical difficulties, such as divided by small numbers. Both the gravity and interfacial friction terms need such treatment. Assuming the gas phase is absent, the gravity term for the gas phase momentum equation is discretized as,

$$
g_{x, i+1 / 2}=\frac{0.5\left(\alpha_{i} \rho_{g, i} g_{x, i}+\alpha_{i+1} \rho_{g, i+1} g_{x, i+1}\right)}{\alpha_{i+1 / 2}^{*} \rho_{g, i+1 / 2}}
$$

in which, $\rho_{g, i+1 / 2}=0.5\left(\rho_{g, i}+\rho_{g, i+1}\right)$ is the averaged gas phase density on cell edge. The treated cell edge void fraction is defined as,

$$
\alpha_{i+1 / 2}^{*}=\left\{\begin{array}{lr}
0.5\left(\alpha_{i}+\alpha_{i+1}\right) & \text { if } 0.5\left(\alpha_{i}+\alpha_{i+1}\right)>\alpha_{\min } \\
\alpha_{\min } & \text { otherwise }
\end{array}\right.
$$

This treated cell edge void fraction is also used to calculate the interfacial drag coefficient (Equations (6) and (7)), as well as in the denominator of the interfacial drag terms in the momentum equations. The liquid phase can be treated similarly when it is absent. We have found 
that this treatment is successful in dealing with the singularity issue. In addition, we have also found that, without this proper treatment, the velocity of the absent phase becomes non-physically large and causes other non-physical numerical behaviors, such as artificial pressure jump.

\subsection{Time integration schemes}

In this section, the time integration schemes will be briefly discussed. Our efforts have been focused on the fully implicit time integration schemes. For the general $\theta$ method, the equation system presented in section 2 could be semi-discretized as,

$$
\frac{\boldsymbol{X}\left(\boldsymbol{U}^{n+1}\right)-\boldsymbol{X}\left(\boldsymbol{U}^{n}\right)}{\Delta t}+\theta \boldsymbol{g}\left(\boldsymbol{U}^{n+1}\right)+(1-\theta) \boldsymbol{g}\left(\boldsymbol{U}^{n}\right)=0
$$

in which, $\Delta t$ is time step size, and superscripts $n$ and $n+1$ denote the old time step and the current time step, respectively. $\boldsymbol{X}=\left[(1-\alpha) \rho_{l}, \alpha \rho_{g}, u_{l}, u_{g}\right]^{\mathrm{T}}$, which is an implicit function of the unknown variable vector, $\boldsymbol{U}\left(=\left[p, \alpha, u_{l}, u_{g}\right]^{\mathrm{T}}\right) \cdot \boldsymbol{g}(\boldsymbol{U})$ represents all non-transient terms in the equation system, including flux terms and source terms. It is noted that the final discretized form is nonlinear functions of the unknown vector $\boldsymbol{U}$, e.g., $\boldsymbol{F}(\boldsymbol{U})=0$. For the $\theta$ parameter, it takes a value of 1 for the time integration scheme to be first-order Backward Euler scheme, and 0.5 for it to be the standard Crank-Nicolson scheme. Using the fully implicit second-order BDF2 scheme, the equation system is semi-discretized as,

$$
\frac{\boldsymbol{X}\left(\boldsymbol{U}^{n+1}\right)-\frac{4}{3} \boldsymbol{X}\left(\boldsymbol{U}^{n}\right)+\frac{1}{3} \boldsymbol{X}\left(\boldsymbol{U}^{n-1}\right)}{\Delta t}+\frac{2}{3} \boldsymbol{g}\left(\boldsymbol{U}^{n+1}\right)=0
$$

Note that, neither the standard Crank-Nicolson scheme nor the BDF2 scheme is strongly stability preserving time integration scheme. However, for most of the applications we are interested in, these methods give satisfactory results, and the unstable behavior can be controlled by reducing the time step size. 


\subsection{Jacobian-free Newton Krylov method}

The resulted discretized nonlinear equations from the implicit method require an iterative method to solve. In this work, we use the JFNK method, which has proved its capability in many disciplines and has been used in our previous work in solving two-phase flow problems. In this subsection, the JFNK method to solve nonlinear system is briefly discussed. For a discretized nonlinear system, such as the discretized two-phase equations we are interested in, one solves,

$$
\boldsymbol{F}(\boldsymbol{U})=0
$$

for the discretized unknown vector, $\boldsymbol{U}=\left[\ldots, p_{i}, \alpha_{i}, u_{l, i+1 / 2}, u_{g, i+1 / 2}, \ldots\right]^{\mathrm{T}}$ in our two-phase flow problems. Thus, Equation (20) represents the $N$ (number of degree of freedom) discretized nonlinear equations. Using the $i^{\text {th }}$ cell and $(i+1 / 2)^{\text {th }}$ cell edge and BDF1 method as an example, the four semi-discretized nonlinear equations are:

$$
\begin{gathered}
\frac{\left(1-\alpha_{i}^{n+1}\right) \rho_{l}\left(p_{i}^{n+1}\right)-\left(1-\alpha_{i}^{n}\right) \rho_{l}\left(p_{i}^{n}\right)}{\Delta t}+\frac{\left[(1-\alpha) \rho_{l} u_{l}\right]_{i+1 / 2}^{*, n+1}-\left[(1-\alpha) \rho_{l} u_{l}\right]_{i-1 / 2}^{*, n+1}}{\Delta x}=0 \\
\frac{\alpha_{i}^{n+1} \rho_{g}\left(p_{i}^{n+1}\right)-\alpha_{i}^{n} \rho_{g}\left(p_{i}^{n}\right)}{\Delta t}+\frac{\left(\alpha \rho_{g} u_{g}\right)_{i+1 / 2}^{*, n+1}-\left(\alpha \rho_{g} u_{g}\right)_{i-1 / 2}^{*, n+1}}{\Delta x}=0 \\
\begin{array}{c}
\frac{u_{l, i+1 / 2}^{n+1}-u_{l, i+1 / 2}^{n}}{\Delta t}+\left(u_{l} \frac{\partial u_{l}}{\partial x}\right)_{i+1 / 2}^{n+1}+\frac{1}{\rho_{l, i+1 / 2}^{n+1}} \frac{p_{i+1}^{n+1}-p_{i}^{n+1}}{\Delta x}-g_{x, i+1 / 2}^{n+1} \\
-\frac{C_{i}^{n+1}}{\left(1-\alpha_{i+1 / 2}^{*, n+1}\right) \rho_{l, i+1 / 2}^{n+1}}\left(u_{g, i+1 / 2}^{n+1}-u_{l, i+1 / 2}^{n+1}\right)\left|u_{g, i+1 / 2}^{n+1}-u_{l, i+1 / 2}^{n+1}\right|=0 \\
\frac{u_{g, i+1 / 2}^{n+1}-u_{g, i+1 / 2}^{n}+\left(u_{g} \frac{\partial u_{g}}{\partial x}\right)_{i+1 / 2}^{n+1}}{\Delta t}+\frac{1}{\rho_{g, i+1 / 2}^{n+1}} \frac{p_{i+1}^{n+1}-p_{i}^{n+1}}{\Delta x}-g_{x, i+1 / 2}^{n+1} \\
-\frac{C_{i}^{n+1}}{\alpha_{i+1 / 2}^{*, n+1} \rho_{g, i+1 / 2}^{n+1}}\left(u_{l, i+1 / 2}^{n+1}-u_{g, i+1 / 2}^{n+1}\right)\left|u_{l, i+1 / 2}^{n+1}-u_{g, i+1 / 2}^{n+1}\right|=0
\end{array}
\end{gathered}
$$


The flux terms in the discretized mass equations, (21) and (22), velocity advection terms and sources terms in the discretized momentum equations, (23) and (24), are described in subsections 3.1 and 3.2.

The solution to the nonlinear system is obtained by iteratively solving a series of Newton's linear correction equations,

$$
\boldsymbol{J}^{k} \delta \boldsymbol{U}^{k}=-\boldsymbol{F}\left(\boldsymbol{U}^{k}\right)
$$

where, $\boldsymbol{J}^{k}$ is the Jacobian matrix; $\boldsymbol{U}^{k}$ is the $k^{\text {th }}$ nonlinear step solution; and $\delta \boldsymbol{U}^{k}$ is the correction vector. In the JFNK solution framework, the linear equations system shown in equation (25), could be effectively solved with a Krylov's method. In the Krylov's method, only a matrix-vector product is required. The matrix-vector product can be approximated as,

$$
\boldsymbol{J}^{k} \boldsymbol{v} \approx \frac{\boldsymbol{F}\left(\boldsymbol{U}^{k}+\epsilon \boldsymbol{v}\right)-\boldsymbol{F}\left(\boldsymbol{U}^{k}\right)}{\epsilon}
$$

in which, $v$ is the Krylov vector and $\epsilon$ is a perturbation parameter. After the correction vector, $\delta \boldsymbol{U}^{k}$, is solved from the linear system, the $(k+1)^{\text {th }}$ nonlinear step solution could be updated as,

$$
\boldsymbol{U}^{k+1}=\boldsymbol{U}^{k}+\delta \boldsymbol{U}^{k}
$$

One of the advantages to use the JFNK method is that the explicit form of the Jacobian matrix could be avoided. The derivation and code implementations of analytical Jacobian matrix could a cumbersome and error-prone task for two-phase flow system analysis codes, since many thermalhydraulics correlations have quite complicated forms. In our implementation of the NewtonKrylov method, the scientific computational toolkit PETSc [20] is used to solve the discretized nonlinear fluid equations. It is important to point out that, JFNK method generally needs a good preconditioning scheme to be effective and efficient. There are different ways to precondition the JFNK method, which were reviewed in Ref. [10]. In our work, the preconditioning matrix is obtained by the default finite differencing method provided in the PETSc package. 


\section{NUMERICAL TESTS}

In this section, the JFNK method along with the high-resolution spatial discretization and fully implicit time integration schemes will be tested with several hydrodynamic two-phase problems with the phase appearance/disappearance phenomena considered. These tests, including a linear advection problem, an oscillation manometer problem, and a sedimentation problem, will be used to test the numerical discretization and time integration schemes as well as the robustness of the JFNK method in solving two-phase problems.

\subsection{Convergence study}

In this subsection, a mesh refinement study was performed to verify the order of accuracy of the high-resolution spatial discretization scheme. The study was performed using a void fraction linear advection problem with a spatially continuous initial sine distribution. The parameters and initial/boundary conditions are summarized in Table 1. The sine profile of the void fraction simply advects along the pipe length, and repeats itself as periodic boundary conditions are used. The mesh refinement study was performed by fixing the CFL number $(\mathrm{CFL}=0.5)$, while the mesh numbers were sequentially doubled from 40 to 640 , and the BDF2 method was used. The L1 norm of the numerical error follows the definition given in [21]:

$$
\|E\|_{1}=\Delta x \sum_{i=1}^{N}\left|\tilde{u}_{i}-u_{\text {exact }}\left(x_{i}\right)\right|
$$

in which, $\Delta x$ is the cell size, $N$ is the total number of cells, $\tilde{u}$ is the numerical solution, and $u_{\text {exact }}$ is the exact solution. The numerical errors and the convergence rate are summarized in Table $\mathbf{2}$. As expected, for spatially smooth solutions, the high-resolution spatial scheme used in this work provides a $2^{\text {nd }}$ order of accuracy. 
Table 1. Parameters, initial and boundary conditions for the mesh refinement test

\begin{tabular}{lll}
\hline Parameter & Value & Unit \\
\hline Pipe length $(L)$ & 1 & {$[\mathrm{~m}]$} \\
Gravity $\left(g_{x}\right)$ & 0.0 & {$\left[\mathrm{~m} / \mathrm{s}^{2}\right]$} \\
\hline$u_{l, \text { init }}$ & 1 & {$[\mathrm{~m} / \mathrm{s}]$} \\
$u_{g, \text { init }}$ & 1 & {$[\mathrm{~m} / \mathrm{s}]$} \\
$\alpha_{g, \text { init }}$ & $0.5+0.2 \sin (2 \pi x / L)$ & \\
$p_{\text {init }}$ & $10^{5}$ & {$[\mathrm{~Pa}]$} \\
\hline$\alpha_{g, \text { inlet }}$ & periodic B.C. & \\
$p_{\text {inlet }}$ & periodic B.C. & \\
$p_{\text {outlet }}$ & periodic B.C. & \\
\hline
\end{tabular}

Table 2. Convergence rate of the mesh refinement test

\begin{tabular}{llll}
\hline $\boldsymbol{N}_{\text {cell }}$ & $\boldsymbol{\Delta} \boldsymbol{x}\left(=\boldsymbol{L} / \boldsymbol{N}_{\text {cell }}\right)$ & $\boldsymbol{L}$-1 Norm & Rate \\
\hline 40 & $2.5000 \mathrm{E}-02$ & $5.0844 \mathrm{E}-03$ & - \\
80 & $1.2500 \mathrm{E}-02$ & $1.2834 \mathrm{E}-03$ & 1.99 \\
160 & $6.2500 \mathrm{E}-03$ & $3.3928 \mathrm{E}-04$ & 1.92 \\
320 & $3.1250 \mathrm{E}-03$ & $8.7250 \mathrm{E}-05$ & 1.96 \\
640 & $1.5625 \mathrm{E}-03$ & $2.1763 \mathrm{E}-05$ & 2.00 \\
\hline
\end{tabular}

\subsection{Linear advection problem}

In nuclear reactor analyses, accurate tracking of scalar quantities, such as boron solute and void fraction distributions in the reactor systems is essential to perform accurate neutronics and thermal-hydraulics coupling calculations. However, in most system analysis codes, due to excessive numerical diffusions from low-order numerical schemes, undesirable less accurate results are often obtained.

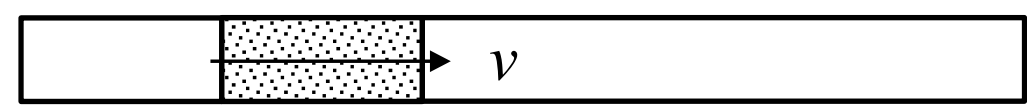

\section{Figure 2, Schematic drawing of the linear advection test problem.}

The linear advection problem describes the advection of scalar quantities with the flow field. In this advection test problem, a $1 \mathrm{~m}$ long pipe is initially filled with two-phase mixtures with $\alpha=$ 1.0, 0.0, and 1.0 in three consecutive sections, moving at the same speed of $1 \mathrm{~m} / \mathrm{s}$, which is 
schematically shown in Figure 2. The pipe is frictionless, and a same pressure is given at the inlet (left) and the outlet (right) boundaries. The parameters for this test are summarized in Table 3. Ideally, the three columns of two-phase mixture would simply advect with their initial velocity and the sharp void fraction interfaces would maintain. Although the test presented here is physically simple, the numerical challenges for this problem are multifold. These include: 1) capturing of sharp spatial discontinuity in solutions; 2) numerical singularity due to phase appearance/disappearance; and 3) stability control for the combination of high-resolution spatial discretization and high-order fully implicit schemes.

In this test, cell numbers of 100 and 400 were used, with a time step $\Delta t=10^{-3} \mathrm{~s}$, corresponding to $\mathrm{CFL}=0.1$ for 100 cells and $\mathrm{CFL}=0.4$ for 400 cells. In order to demonstrate the advantages of using high-resolution spatial discretization and high-order implicit time integration schemes, numerical results using first-order spatial discretization and first-order BDF1 methods were also obtained for comparison purpose. The numerical results of the simulations are shown in Figure 3. It can be observed that, the numerical results from low-order method using 400 cells are only comparable to that from high-order method using 100 cells. Such a comparison between the highorder and low-order methods clearly shows that the high-order methods give much less numerical diffusion. A convergence study was not performed for this test problem, because the expected order of accuracy in general cannot be obtained when a discontinuous solution exists (see section 8.7 of [21]). Based on the spatial and temporal schemes used in our work, the JFNK method is able to correctly calculate the void fraction distribution, even when $\alpha=0$ or 1 . However, also from Figure 3, it can be observed that the high-order method (using 400 cells) gives negative void fraction values (undershoot). This is due to the fact that the BDF2 scheme is not a strongly stability preserving method. Although the magnitude of undershoot is small (less than $10^{-6}$ ) and the JFNK method could continue well, it may cause serious issue (such as nonphysical solutions due to the negative void fraction value) when realistic closure laws are applied. This issue can be 
resolved by reducing the time step size (e.g., from $10^{-3} s$ to $5 \times 10^{-4} s$, CFL reduced from 0.4 to 0.2) or by using the more stable BDF1 method with the results shown in Figure 4. The undershoot behavior of the BDF2 method indicates that, if a high-order fully implicit scheme is desirable, a time step control mechanism in a production code will be necessary. However, since there is no theoretical basis for such a control mechanism, a heuristic approach would be used to determine the appropriate time step size. For example, an overshoot/undershoot detection could be performed to determine if the current time step size is acceptable.

Table 3. Parameters, initial and boundary conditions for the linear advection test problem with phase appearance/disappearance

\begin{tabular}{|c|c|c|}
\hline Parameter & Value & Unit \\
\hline Pipe length $(L)$ & 1 & {$[m]$} \\
\hline Gravity $\left(g_{x}\right)$ & 0.0 & {$\left[\mathrm{~m} / \mathrm{s}^{2}\right]$} \\
\hline$u_{l, \text { init }}$ & 1 & {$[\mathrm{~m} / \mathrm{s}]$} \\
\hline$u_{g, \text { init }}$ & 1 & {$[\mathrm{~m} / \mathrm{s}]$} \\
\hline$\alpha_{g, \text { init }}$ & $\left\{\begin{array}{rr}0.0 & \text { if } 0.2 \leq x \leq 0.4 \\
1.0 & \text { otherwise }\end{array}\right.$ & \\
\hline$p_{\text {init }}$ & $10^{5}$ & {$[\mathrm{~Pa}]$} \\
\hline$\alpha_{g, \text { inlet }}$ & periodic B.C. & \\
\hline$p_{\text {inlet }}$ & periodic B.C. & \\
\hline$p_{\text {outlet }}$ & periodic B.C. & \\
\hline$N_{\text {cell }}$ & $100-400$ & \\
\hline$\Delta t$ & $10^{-3}$ & {$[s]$} \\
\hline$N_{\text {timestep }}$ & $10^{2}$ & \\
\hline
\end{tabular}




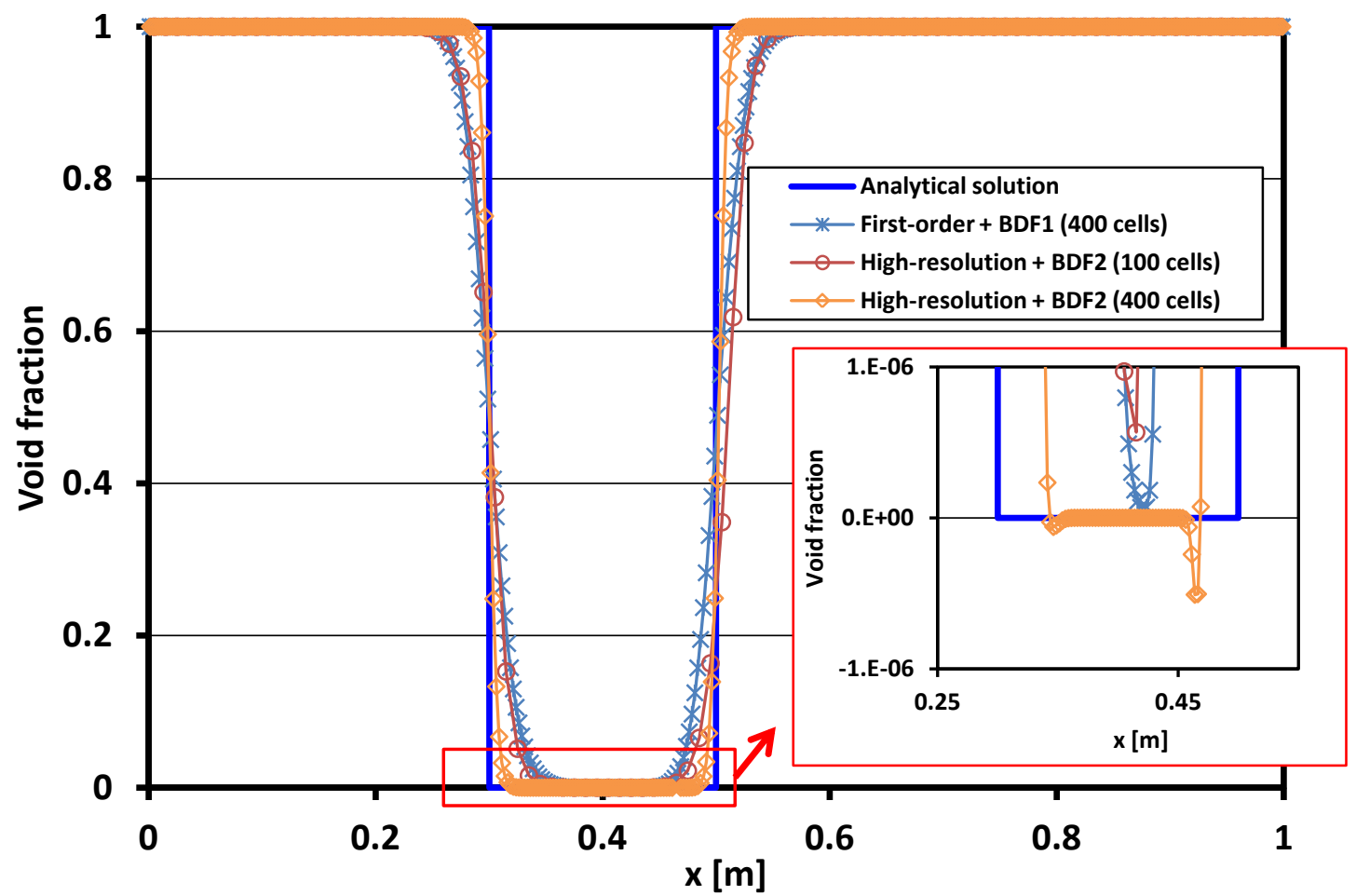

Figure 3. Void fraction distribution for the linear advection test problem with phase appearance/disappearance, with $\left(N_{\text {cell }}=100, \Delta t=10^{-3} \mathrm{~s}, \mathrm{CFL}=0.1, \mathrm{BDF} 2\right)$ and $\left(N_{\text {cell }}=400, \Delta t=\right.$ $\left.10^{-3} \mathrm{~s}, \mathrm{CFL}=0.4, \mathrm{BDF} 1 / \mathrm{BDF} 2\right)$, and $N_{\text {timestep }}=10^{2}$

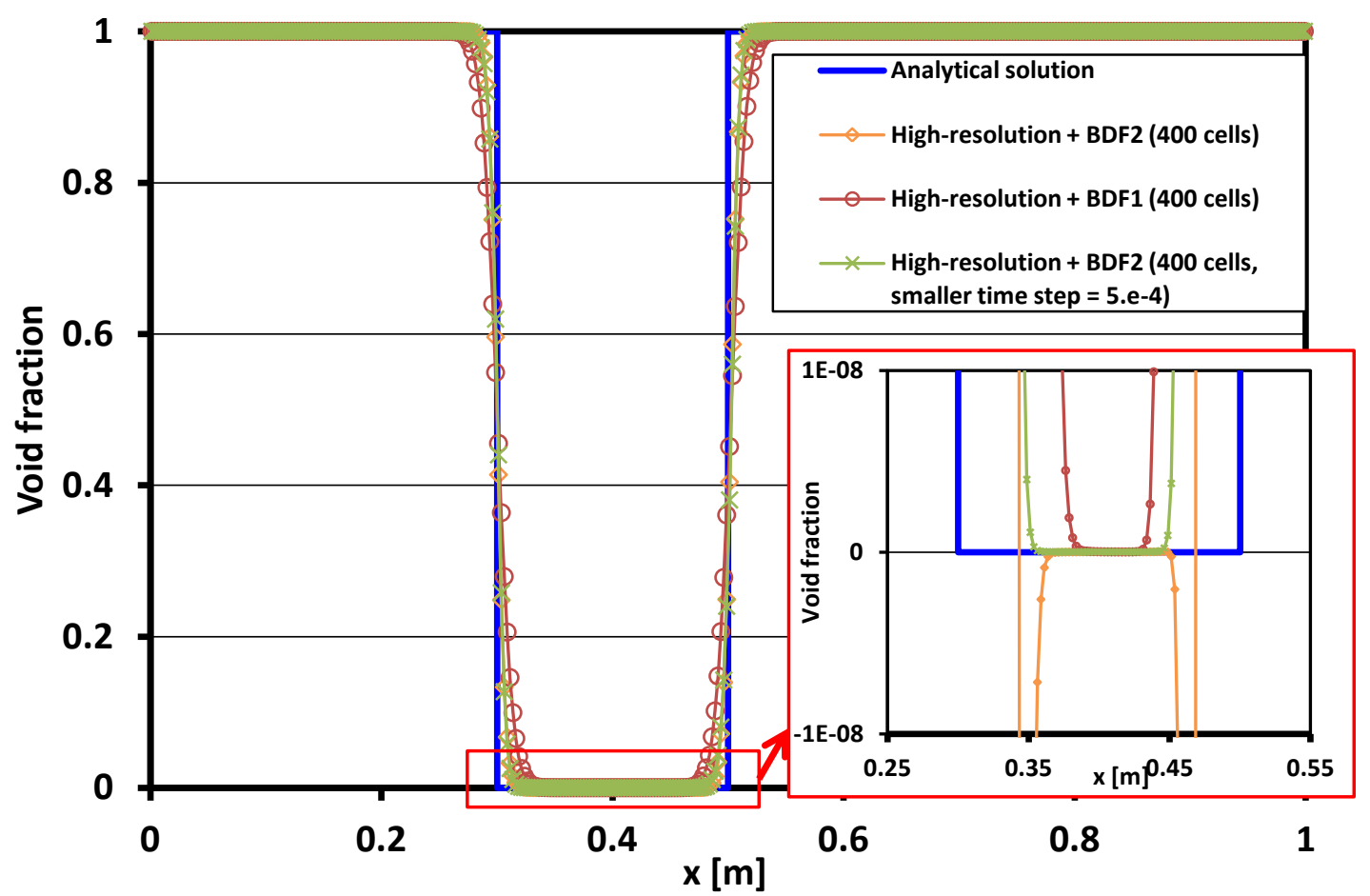

Figure 4. Void fraction distribution for the linear advection test problem with phase appearance/disappearance, with $\left(N_{\text {cell }}=400, \Delta t=10^{-3} s, \mathrm{CFL}=0.4, \mathrm{BDF} 1 / \mathrm{BDF} 2, N_{\text {timestep }}=10^{2}\right)$, and $\left(N_{\text {cell }}=400, \Delta t=5 \times 10^{-4} \mathrm{~s}, \mathrm{CFL}=0.2, \mathrm{BDF} 2, N_{\text {timestep }}=2 \times 10^{2}\right)$ 


\subsection{Oscillating manometer problem}

The oscillating manometer test problem was originally proposed by Ransom as a numerical benchmark test for two-phase flows [22]. The problem consists of a "U" tube manometer, which is partially filled with liquid and vapor (see Figure 5 (left) for the schematic drawing). This problem provides a test for the modeling of inertia and body force effects. In addition, the phase appearance/disappearance phenomenon exists and is similar to that in the linear advection problem. In the numerical test, a straight pipe with different gravity constants is used to simulate the manometer problem, similar to the problem setup as in [22] and [23]. A schematic drawing and nodalization of the manometer problem is shown in Figure 5 (right). Parameters, boundary and initial conditions for the numerical tests are summarized in Table 4.
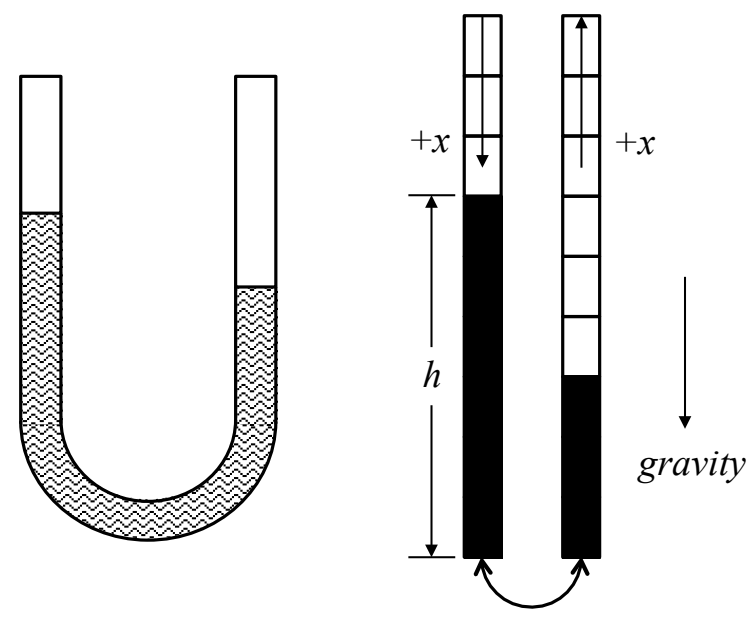

Figure 5, Schematic drawing (left) and nodalization (right) of the oscillating manometer problem.

This problem has been numerically studied in several research works and numerical/analytical results were provided. García Cascales's [24] tried to use several different spatial discretization schemes to solve the manometer problem, however the problem could neither be solved, nor be solved correctly. Satisfactory numerical results were obtained in a later work done by Paillere and his coworkers [8]. This problem has also been included in the RELAP5-3D code developmental 
assessment [23]. With specially designed level tracking scheme, the numerical results are accurate compared to the analytical solutions. Another numerical test of the manometer problem could be found in Städtke's work [17]. Based on the commonly used single-pressure two fluid two-phase flow model, Städtke developed a hyperbolic model by carefully examining and modifying the interfacial momentum coupling terms. Second-order accuracy Godunov type of finite volume method and implicit time integration scheme were used to solve the two-phase problem. The numerical test showed good results from the comparison between numerical results and analytical solutions of liquid phase velocity. However, to achieve hyperbolicity, the conservative equations have very complex forms (as an example, interested readers are referred to see equation 5.21 in ref. [17] for the nonviscous interfacial momentum term). The scheme used to evaluate the cell interface numerical fluxes is also cumbersome, which requires complex matrix and vector operations.

Table 4. Parameters, initial and boundary conditions for the oscillating manometer problem

\begin{tabular}{|c|c|c|}
\hline Parameter & Value & Unit \\
\hline Pipe length $(L)$ & 20 & {$[m]$} \\
\hline Gravity $\left(g_{x}\right)$ & $\left\{\begin{array}{cc}9.81 & 0 \leq x<10 \\
0 & x=10 \\
-9.81 & 10<x \leq 20\end{array}\right.$ & {$\left[\mathrm{m} / \mathrm{s}^{2}\right]$} \\
\hline$u_{l, \text { init }}$ & -1.0 & {$[\mathrm{~m} / \mathrm{s}]$} \\
\hline$u_{g, \text { init }}$ & -1.0 & {$[\mathrm{~m} / \mathrm{s}]$} \\
\hline$\alpha_{g, \text { init }}$ & $\left\{\begin{array}{cr}0.0 & \text { if } 5 \leq x \leq 15 \\
1.0 & \text { otherwise }\end{array}\right.$ & \\
\hline$p_{\text {init }}$ & $10^{5}+\rho_{\text {init }}|g| h$ & {$[P a]$} \\
\hline$\alpha_{g, l e f t, b c}$ & 1.0 & \\
\hline$\alpha_{g, r i g h t, b c}$ & 1.0 & \\
\hline$p_{\text {left }, b c}$ & $10^{5}$ & {$[P a]$} \\
\hline$p_{r i g h t, b c}$ & $10^{5}$ & {$[\mathrm{~Pa}]$} \\
\hline$N_{\text {cell }}$ & 200 & \\
\hline$\Delta t$ & $10^{-2}$ & {$[s]$} \\
\hline$N_{\text {timestep }}$ & 1500 & \\
\hline
\end{tabular}

In this study, numerical results of the manometer test problem were obtained, using 200 cells, a time step of $10^{-2} s(\mathrm{CFL}=0.1)$, total number of time steps of 1500 . Numerical results of the 
liquid phase velocity at the bottom of the "U" tube, the liquid level in the left leg of the manometer, and the void fraction spatial distribution are plotted in Figure 6, Figure 7, and Figure 8 , respectively. The liquid level was determined by the location of $\alpha=0.5$, which is done with interpolation. The numerical results from the high-resolution spatial discretization and the BDF2 time integration schemes agree with the analytical solutions very well. As expected, the numerical results from first-order spatial discretization and BDF1 time integration schemes display numerical damping effect due to excessive numerical diffusion.

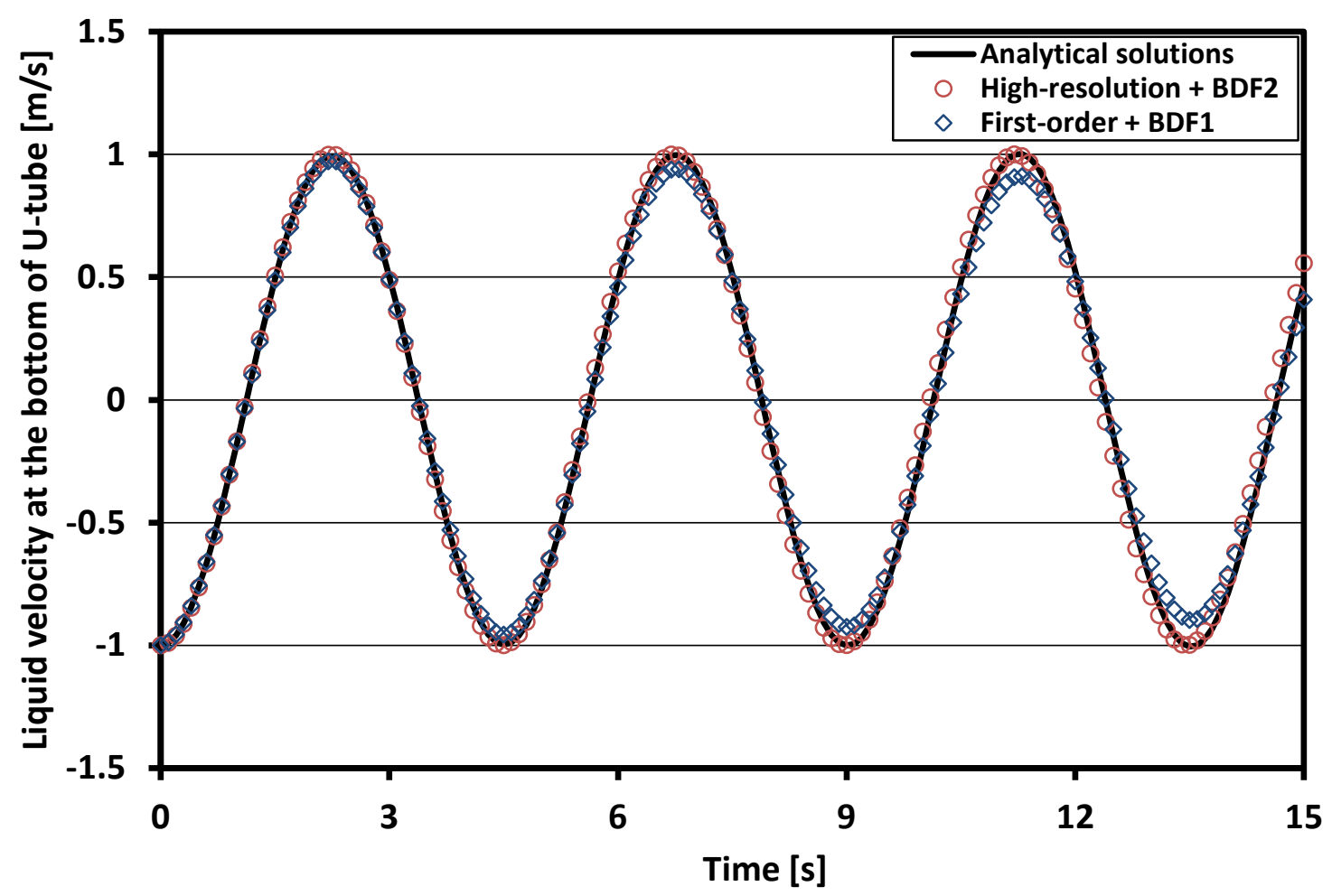

Figure 6, Liquid velocity at the bottom of the manometer, with $N_{\text {cell }}=200, \Delta t=10^{-2} \mathrm{~s}, \mathrm{CFL}=0.1$, and $N_{\text {timestep }}=1500$. 


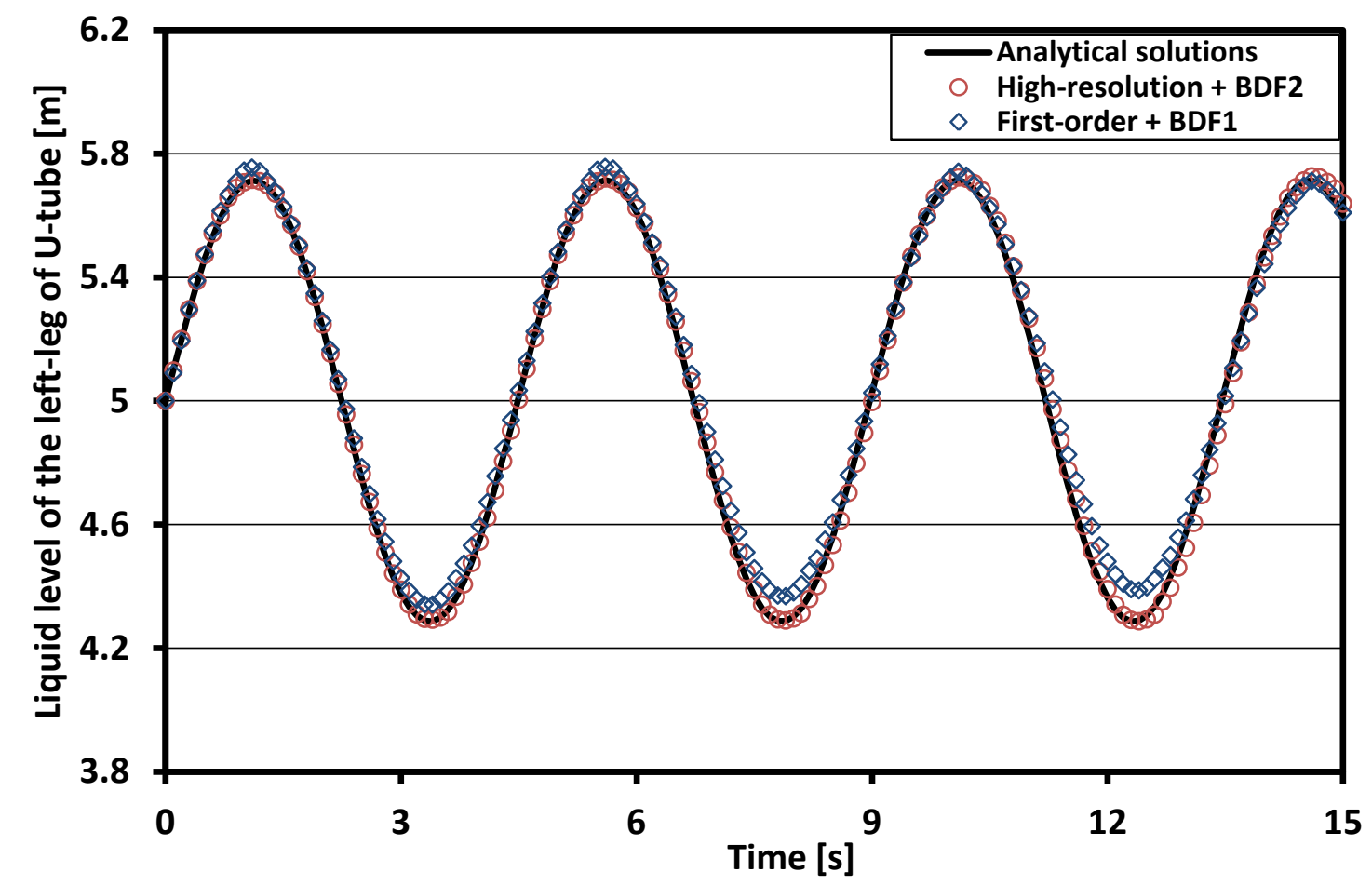

Figure 7, Height of the liquid level on the left leg of the manometer, with $N_{\text {cell }}=200, \Delta t=10^{-2} s$, $\mathrm{CFL}=0.1$, and $N_{\text {timestep }}=1500$. The liquid level is determined by $\alpha=0.5$ interpolation.

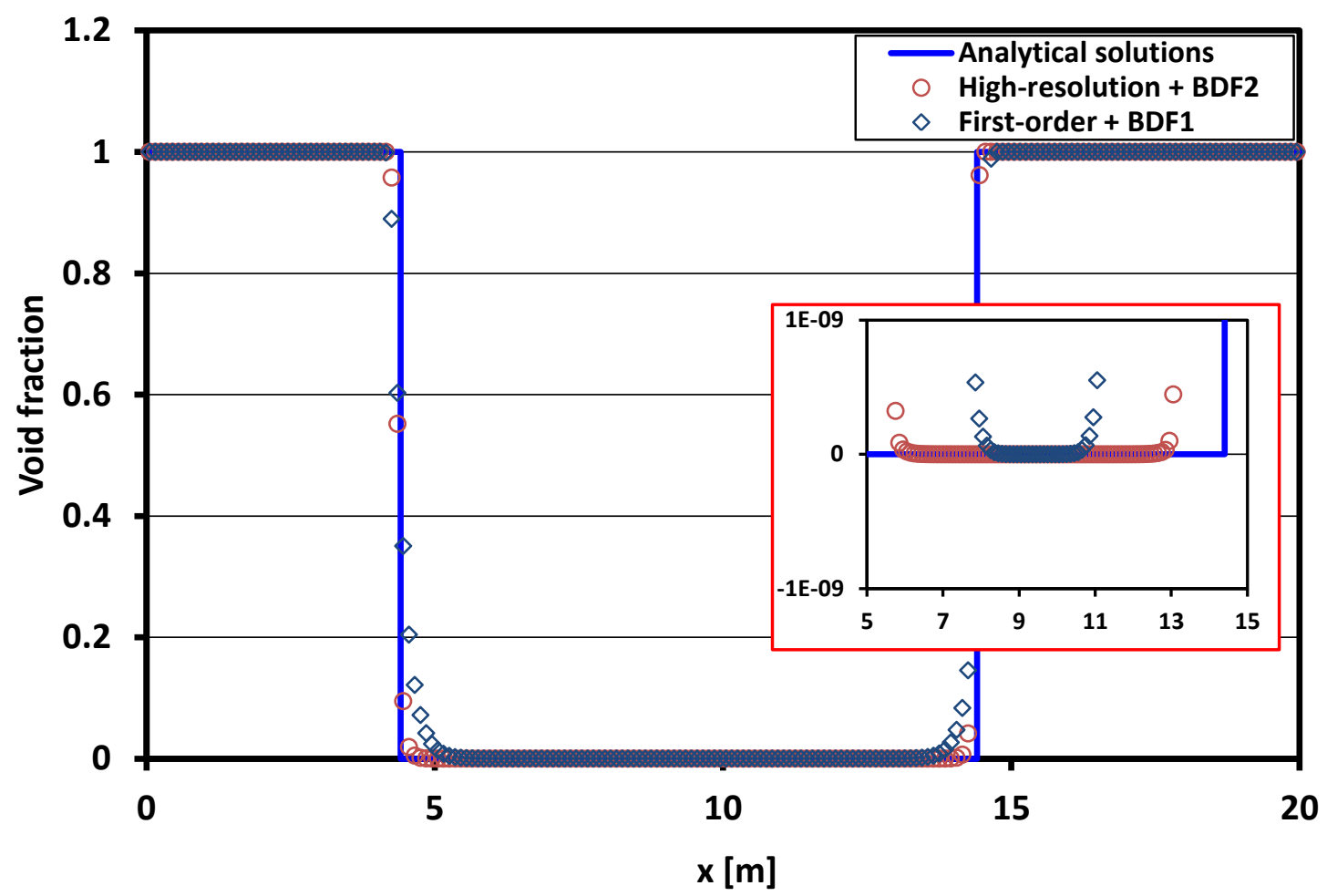

Figure 8, The distribution of void fraction at the end of simulation, $\mathrm{t}=15 \mathrm{~s}$, with $N_{\text {cell }}=200, \Delta t=$ $10^{-2} s, \mathrm{CFL}=0.1$, and $N_{\text {timestep }}=1500$. The liquid level is determined by $\alpha=0.5$ interpolation. 


\subsection{Sedimentation problem}

The sedimentation problem is a test problem originally proposed by Youngs [22] for testing the phase separation due to gravity. The test consists of a vertical pipe initially filled with a twophase mixture having a uniform void fraction $\alpha=0.5$. Due to the gravity effect, the two phases will eventually separate into two single-phase zones with the heavier liquid phase settling down at the bottom, and the lighter phase at the top of the pipe. A schematic drawing of the sedimentation problem is shown in Figure 9. The problem was originally proposed to use two fluids with different however very close densities (0.999 compared to 1.000). In many simulations, more realistic values have been used to simulate a water/vapor (gas) separation [17, 24-26]. A similar configuration is used in this study, with the parameters summarized in Table $\mathbf{5}$. The problem is designed to test the gravity effect, as well as the flow model's capability in simulating two-phase counter current flow. Besides, the sedimentation problem also casts a challenge for the numerical method in solving the singular two-phase flow problem with phase appearance/disappearance. In this test, the singularity is dynamically developed from the phase separation process, which is different from that of the advection problem presented in section 4.1.

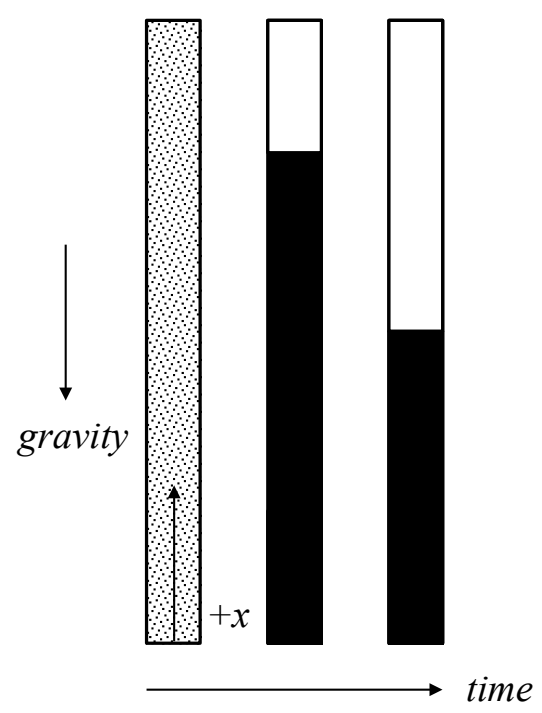

Figure 9, Schematic drawing of the sedimentation test problem. 
Table 5. Parameters, initial and boundary conditions for the sedimentation problem

\begin{tabular}{lll}
\hline Parameter & Value & Unit \\
\hline Pipe length $(L)$ & 2.0 & {$[\mathrm{~m}]$} \\
Gravity $\left(g_{x}\right)$ & -9.81 & {$\left[\mathrm{~m} / \mathrm{s}^{2}\right]$} \\
\hline$u_{\text {l,init }}$ & 0.0 & {$[\mathrm{~m} / \mathrm{s}]$} \\
$u_{g, \text { init }}$ & 0.0 & {$[\mathrm{~m} / \mathrm{s}]$} \\
$\alpha_{g, \text { init }}$ & 0.5 & \\
$p_{\text {init }}$ & $10^{5}$ & {$[\mathrm{~Pa}]$} \\
\hline Inlet & Non-penetrating wall & \\
Outlet & Non-penetrating wall & \\
\hline$N_{\text {cell }}$ & 50 & \\
$\Delta t$ & $5 \times 10^{-3}$ & {$[\mathrm{~s}]$} \\
$N_{\text {timestep }}$ & 2000 & \\
\hline
\end{tabular}

Numerical results were obtained by using 50 cells and a time step size of $1 \times 10^{-2} s(\mathrm{CFL} \approx 0.25)$. Figure 10 shows the transient void fraction distribution along the pipe. Two discontinuous void fronts could be found to move upward and downward, and eventually merged at 10 seconds. Figure 10 is also enlarged into Figure 11 to demonstrate the void fraction distributions near 0 and 1. It can be seen that the phase separation is well captured, and void fractions are well bounded between 0 and 1.

The transient pressure profiles are shown in Figure 12. There are no analytical solutions for the transient pressure distribution and such transient profiles have been rarely reported in existing literatures. In addition, inconsistent pressure distributions have been reported in them. GarcíaCascales [24] obtained two totally different transients of pressure distributions from two different numerical methods, and one of the results seems to be numerically unstable (large spatial pressure oscillation). Coquel et al. [25] only provided a pressure distribution at steady-state conditions. We have found that our results are almost identical to what Städtke have predicted [17], which is somewhat expected since similar interfacial drag terms were used. Without using the numerical 
treatment described in subsection 3.2, an artificial pressure jump was found near the liquid-gas interface. A similar artificial pressure jump is also evident in Munkejord' work (Fig. 4.7 in Ref. [26]), and also in Guillard's work (Fig. 3 in Ref. [27]). A possible reason for such pressure jump was discussed in the work done by Abe et al. [28]. It was concluded in [28] that, the untreated velocity of the absent phase causes artificial pressure jump across the sharp interface, and this velocity should be properly bounded. Paill $\square$ ere et al. [8] also presented a similar concept to bound the velocity of the absent (disappearing) phase. In this work, we found that the numerical treatment proposed in subsection 3.2 works well to remove the artificial pressure jump near the liquid-gas interface. Pressure profiles at 10 seconds were obtained using different cell numbers, ranging from 20 to 1000 . The results are plotted in Figure 13, and it can be observed that all pressure profiles nicely overlap with each other.

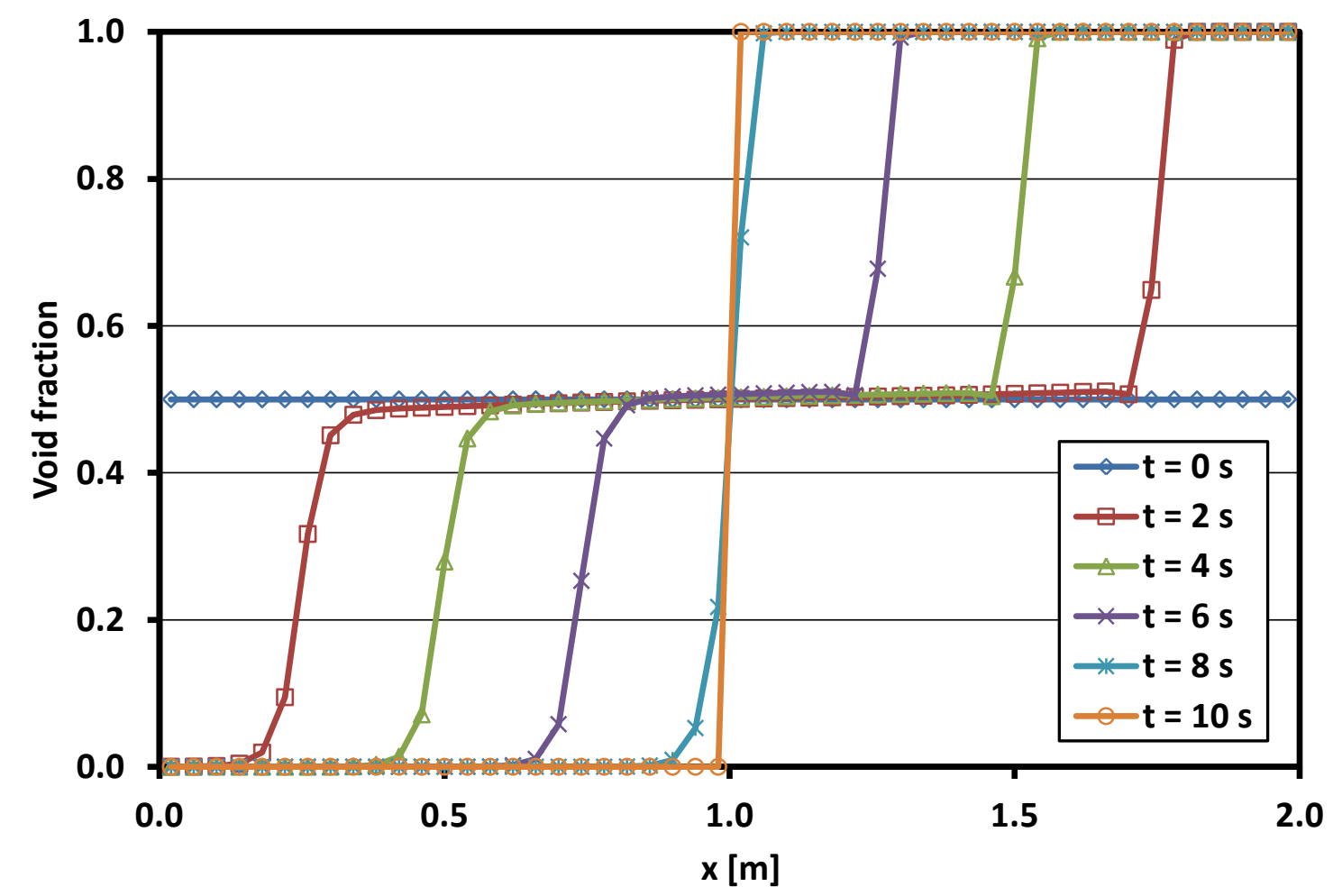

Figure 10, Void fraction distribution at different time for the sedimentation test problem, with $N_{\text {cell }}=50, \Delta t=1 \times 10^{-2} s,(\mathrm{CFL} \approx 0.25)$. 

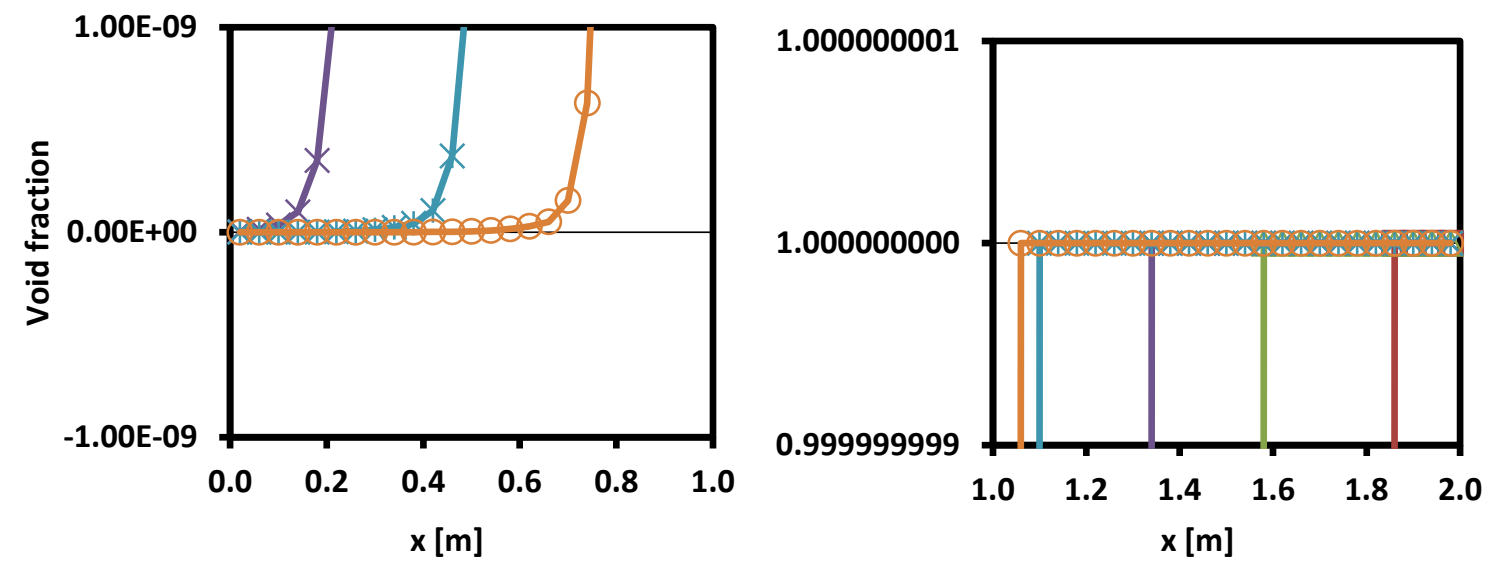

Figure 11, Void fraction distribution at different time for the sedimentation test problem, with $N_{\text {cell }}=50, \Delta t=1 \times 10^{-2} s,(\mathrm{CFL} \approx 0.25)$. Enlarged from Figure 10 for void fraction regions near 0 (left) and 1 (right).

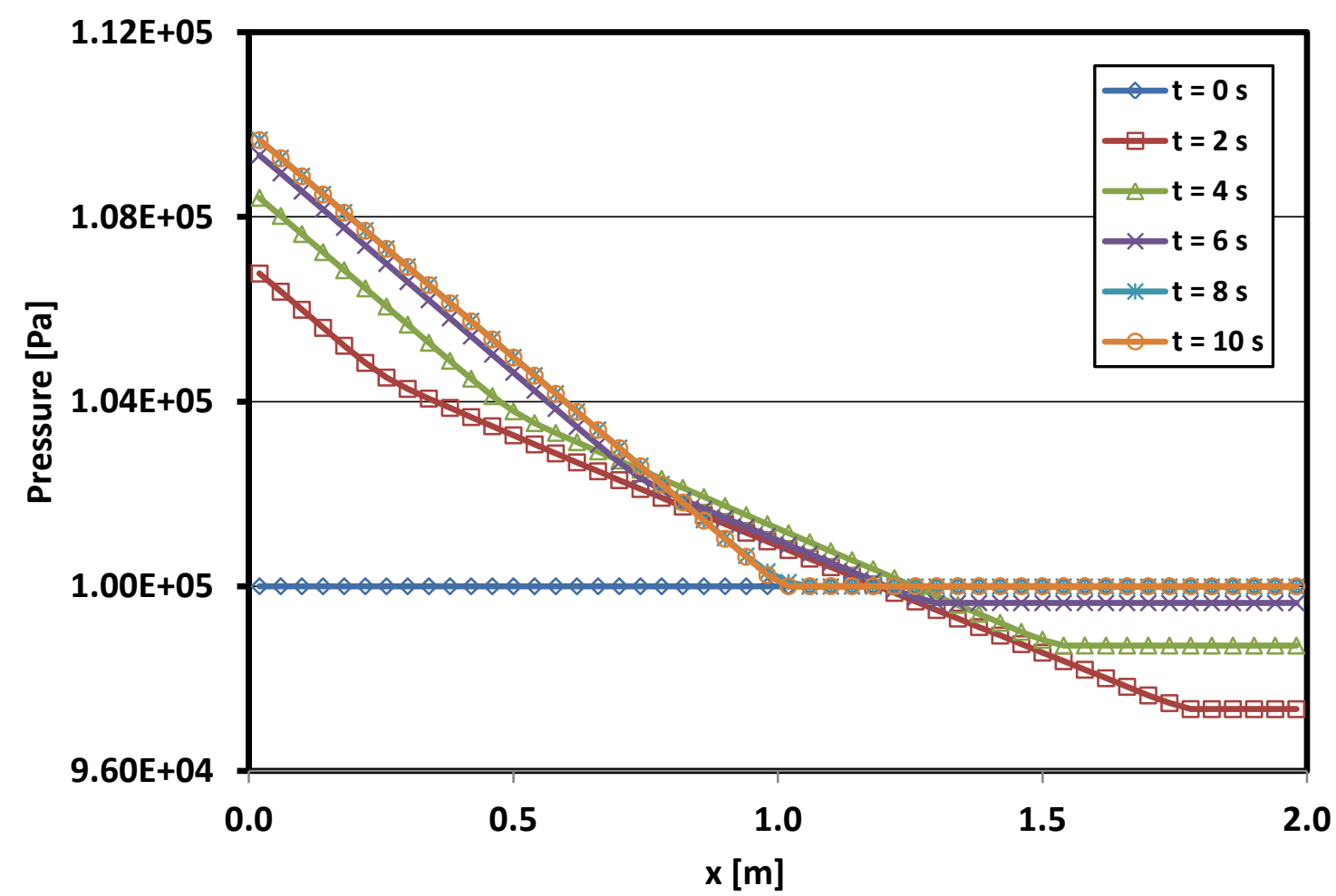

Figure 12, Pressure distribution at different time for the sedimentation test problem, with $N_{\text {cell }}=$ $50, \Delta t=1 \times 10^{-2} s,(\mathrm{CFL} \approx 0.25)$. 


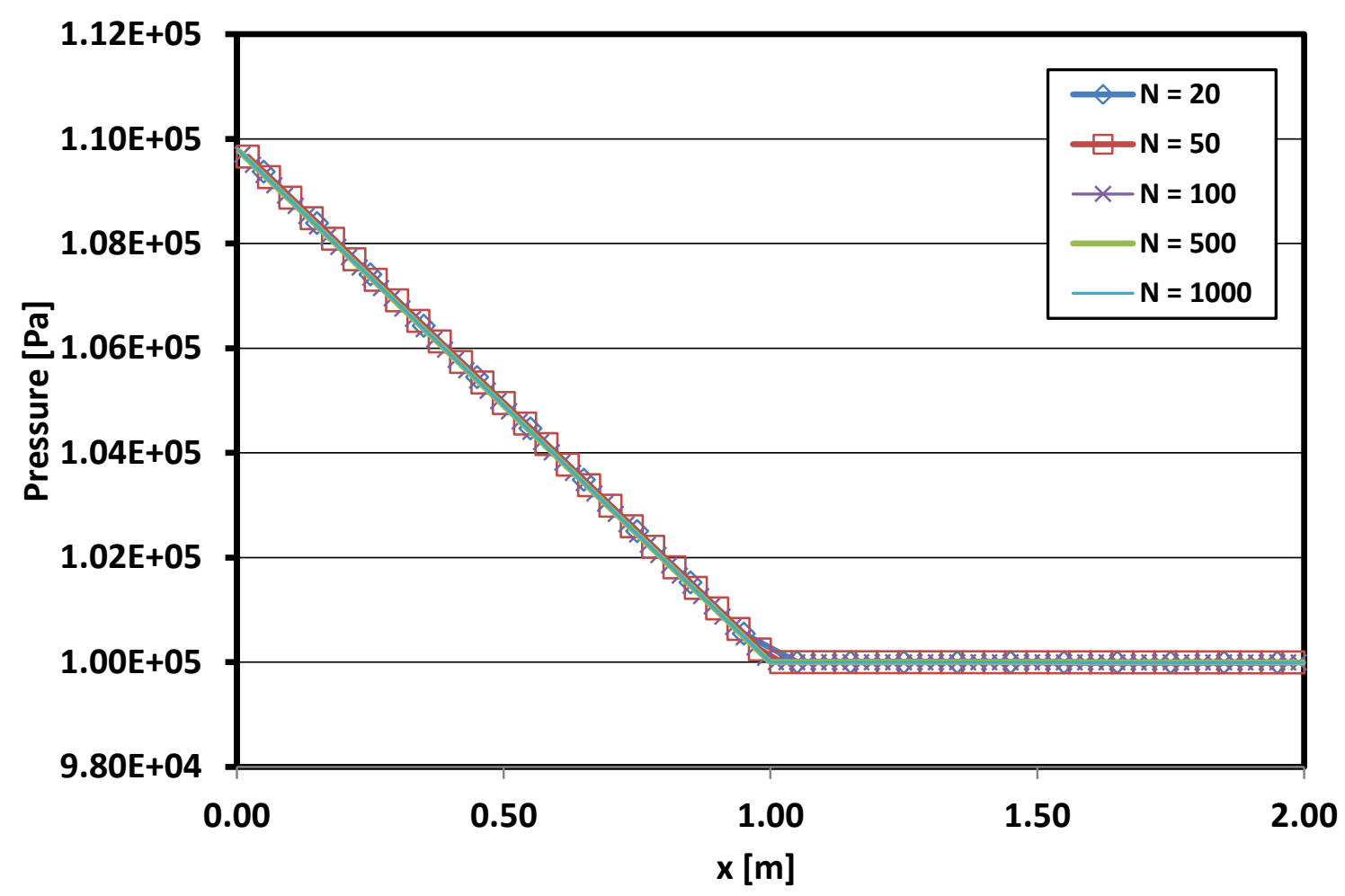

Figure 13, Pressure distribution at the end of the simulation for the sedimentation test problem, with $\Delta t=5 \times 10^{-3} s, N_{\text {timestep }}=2000\left(t_{\text {end }}=10 \mathrm{~s}\right)$, using different numbers of cells, 20, 50, 100; also, with $\Delta t=1 \times 10^{-3} s, N_{\text {timestep }}=10000\left(t_{\text {end }}=10 \mathrm{~s}\right)$, using 500 and 1000 cells.

\section{Conclusions}

In this study, high-resolution spatial discretization and second order fully implicit time integration schemes have been applied to solve one-dimensional two-phase flow problems with phase appearance/disappearance phenomena considered. The discretized nonlinear equations were successfully solved with the JFNK method. By using such a method, the cumbersome derivation and error-prone implementation of analytical Jacobian matrix could be avoided. The combined methods used in this work demonstrated extremely robust and stable behaviors in solving singular problem when one phase vanishes or appears. Numerical treatments were introduced for the momentum equation of the disappearing (absent) phase without the need to use a cut-off value of the void fraction. High-resolution spatial discretization and second-order fully implicit method also demonstrated their capabilities in significantly reducing numerical errors. It is also confirmed 
in the numerical studies that, the second-order fully implicit BDF2 method is not a strongly stability preserving method. This issue can be resolved by reducing time step or by using the more stable BDF1 method. The numerical studies therefore suggest that a time control mechanism would be necessary if such a high-order time integration scheme is desired.

\section{Acknowledgement}

This work is supported by the U.S. Department of Energy, under Department of Energy Idaho Operations Office Contract DE-AC07-05ID14517. Accordingly, the U.S. Government retains a nonexclusive, royalty-free license to publish or reproduce the published form of this contribution, or allow others to do so, for U.S. Government purposes.

\section{References}

1. "RELAP5/MOD3.3 Code Manual Volume I", NUREG/CR- 5535 ed., U.S. Nuclear Regulatory Commission, December, 2001.

2. “TRAC-M/FORTRAN 90 (Version 3.0) Theory Manual”, NUREG/CR-6724 ed., U.S. Nuclear Regulatory Commission, April, 2001.

3. "RELAP5-3D Code Manual Volume I: Code Structure, System Models and Solution Methods", INEEL-EXT-98-00834, Revision 4.0, June 2012.

4. F. Barre, M. Parent, and B. Brun, "Advanced Numerical Methods for Thermalhydraulics", Proceedings of the CSNI Specialist Meeting on Transient Twophase Flow, Aix-en-Provence, France, April 6-8, 1992.

5. D. Bestion, "The Phase Appearance and Disappearance in the CATHARE Code", Trends in Numerical and Physical Modeling for Industrial Multiphase Flows, Cargese, (Corse), France, September 27-29, 2000.

6. F. Cordier, P. Degond, and A. Kumbaro, "Phase Appearance or Disappearance in TwoPhase Flows", Journal of Scientific Computing, Vol. 58, 1, 115-148, 2014. 
7. A. Ashrafizadeh, C.B. Devaud, and N.U. Aydemir, "A Jacobian-free Newton-Krylov Method for Thermalhydraulics Simulations", Int. J. Numer. Meth. Fluids, DOI: 10.1002/fld.3999, 2015.

8. H. Paillere, C. Corre, and J.R. García Cascales, "On the Extension of the AUSM+ Scheme to Compressible Two-fluid Models", Computing Fluids, Vol. 32, 6, 891-916, 2003.

9. C. Frepoli, J. H. Mahaffy, and K. Ohkawa, "Notes on the implementation of a fullyimplicit numerical scheme for a two-phase three-field flow model”, Nuclear Engineering and Design, 225, 191-217 (2003).

10. D. A. Knoll and D. E. Keyes, "Jacobian-free Newton-Krylov Methods: a Survey of Approaches and Applications", J. Comp. Phys., 193, 357-397 (2004).

11. V. A. Mousseau, "Implicitly Balanced Solution of the Two-phase Flow Equations Coupled to Nonlinear Heat Conduction”, J. Comp. Phys., 200, 104-132 (2004).

12. V. A. Mousseau, "Accurate Solution of the Nonlinear Partial Differential Equations from Thermal Hydraulics", Nuclear Technology, 158, 26-35 (2005).

13. V. A. Mousseau, "A Fully Implicit, Second Order in Time, Simulation of a Nuclear Reactor Core", Proceedings of ICONE-14, International Conference on Nuclear Engineering, Miami, Florida, USA, July 17-20, 2006

14. V. A. Mousseau, "A Fully Implicit Hybrid Solution Method for a Two-Phase ThermalHydraulic Model”, Journal of Heat Transfer, 127, $531-539$ (2005)

15. M. A. Pope and V. A. Mousseau "Accuracy and Efficiency of a Coupled Neutronics and Thermal Hydraulics Model" The 12th International Topical Meeting on Nuclear Reactor Thermal Hydraulics (NURETH- 12), Pittsburgh, Pennsylvania, U.S.A., September 30October 4, 2007.

16. D. Bestion, "The physical closure laws in the CATHARE code", Nuclear Engineering and Design, 124, 229 (1990). 
17. H. Städtke, “Gasdynamic Aspects of Two-phase Flow”, Wiley-VCH, 2006.

18. G.S. Stelling and S.P.A. Duinmeijer, "A staggered conservative scheme for every Froude number in rapidly varied shallow water flows", Int. J. Numer. Meth. Fluids, Vol. 43, pp. 1329-1354 (2003).

19. E.F. Toro, "Riemann Solvers and Numerical Methods for Fluid Dynamics", Second Edition, Springer (1999).

20. S. Balay et al., "PETSc Users Manual, Reversion 3.4”, ANL-95/11 (2013).

21. R. J. LeVeque, "Finite-Volume Methods for Hyperbolic Problems", Cambridge University Press, (2004).

22. Numerical Benchmark Test, volume 2 of Multiphase Science and Technology, Edited by G.F. Hewitt, J.M. Delhaye, and N. Zuber, John Wiley \& Sons Ltd., 1987.

23. "RELAP5-3D Code Manual Volume III: Developmental Assessment”, INEEL-EXT-9800834, Revision 4.0, June 2012.

24. J. R. García-Cascales, Conservative numerical schemes for unsteady 1D two-phase flow, PhD thesis, Universidad Politécnica de Valencia, Spain, 2001.

25. F. Coquel et al., A Numerical Method Using Upwind Schemes for the Resolution of Two-Phase Flows, J. Comp. Phys., 136, 272-288 (1997).

26. S. T. Munkejord, "Analysis of the two-fluid model and the drift-flux model for numerical calculation of two-phase flow", PhD Dissertation, Norwegian University of Science and Technology, 2006.

27. H. Guillard and M. Labois, Numerical Modelling of Compressible Two- phase Flows, European Conference on Computational Fluid Dynamics (ECCOMAS CFD 2006), Egmond aan Zee, The Netherlands, September 5-8, 2006.

28. Y. Abe, H. Akimoto, H. Kamo and Y. Murao, "Elimination of numerical pressure spikes induced by two-fluid model", J. Nucl. Sci. Technol., 30, 1212-1224, (1993). 